\title{
The Influence of Infants' Characteristics on Breastfeeding Attitudes among Jordanian Mothers
}

\author{
Ghada Mohammad Abu Shosha \\ Department of Child Health Nursing, Faculty of Nursing, Zarqa University, Zarqa, Jordan \\ Email: ghada abushosha@yahoo.com
}

Received 15 March 2015; accepted 28 March 2015; published 31 March 2015

Copyright () 2015 by author and Scientific Research Publishing Inc.

This work is licensed under the Creative Commons Attribution International License (CC BY). http://creativecommons.org/licenses/by/4.0/

(c) (i) Open Access

\section{Abstract}

Background: Breastfeeding is the desired nutritional method for infants. Some infants' characteristics were found influential to mothers' point of views toward breastfeeding. However, the relationship between infants' characteristics and mothers' attitudes toward breastfeeding is still uncertain. The aim of this study was to assess infants' characteristics and their impact on Jordanian mothers' attitudes toward breastfeeding. Methods: A descriptive cross-sectional design was used. A sample of 130 mothers was selected conveniently from mothers attending four major governmental maternal-child health centers at Zarqa city in Jordan. The Iowa Infant Feeding Attitude Scale along with infants' characteristics data sheet were used for data collection. Results: A more positive attitude toward breastfeeding was manifest (mean 63.5, SD: 4.67). Positive attitudes toward breastfeeding were higher among women who delivered normally than women who delivered by cesarean section $(p=0.040)$. In addition, women who had healthy infants were more likely to have positive attitudes toward breastfeeding compared to women with ill infants $(p=$ 0.021). However, women with preterm deliveries were less likely to have positive attitudes compared to women with full-term deliveries $(p=0.013)$. Likewise, women whose infants admitted to neonatal intensive care unit recorded less positive attitudes toward breastfeeding than women with healthy neonates $(p=0.043)$. Gestational age, admission to neonatal intensive care unit, infant's health status, and type of delivery, were factors influencing mothers' attitudes towards breastfeeding. Conclusions: Jordanian mothers have positive attitudes toward breastfeeding. However, positive attitudes are subject to different infants' characteristics that should be considered while undertaking heath education programs for promoting breastfeeding. More focusing is recommended for women who delivered preterm infants, women who have ill infants, and women who delivered by cesarean section.

\section{Keywords}

Breastfeeding, Attitudes, Infants, Jordan 


\section{Introduction}

Human milk is considered the most ideal and effective natural way for infants' feeding [1]-[3]. Breastfeeding has an impact on a child's survival, health, growth and development. Breast milk contains all nutrients and vitamins that infants need. Furthermore, breast milk provides infants with antibodies against the majority of diseases. Breastfeeding also increases the bonding and attachment between mothers and babies. It also lowers the risk of chronic diseases including obesity, hypertension, diabetes, asthma and leukaemias. It was agreed that infants who were breastfed had better scores on intelligence and behavior tests later of life compared to infants with bottle-feeding [1].

According to the World Health Organization (WHO), the lives of about 220,000 children would be saved every year if they were breastfed within the first hour of birth, continued exclusive breastfeeding for the first six months of life, and given breastfeeding with age appropriate feeding until the age of two years [2]. Islamic instructions encourage mothers to nurse their children as mentioned in the Holy Qur'an, the book of Muslims, "mothers shall nurse their children for two whole years if they wish to complete the period of nursing" [4].

Based on the evidences of the long-term benefits of breastfeeding for infants, mothers, and societies, the WHO and the American Academics of Pediatrics recommend exclusive breastfeeding for the first six months of life [2] [5]. Unfortunately, less than $40 \%$ of infants under six months of age are exclusively breastfed worldwide [2].

In Jordan, infants feeding practices have become worse over time. According to Jordan Department of Statistics, the proportion of infants ranged in age from 0 - 5 months who are not breastfed has increased from $10 \%$ in 2002 to 13\% in 2013. In 2012, the Jordan Department of Statistics reported that only about 23\% of mothers exclusively breastfeed their children during the first six months of age, and only $42 \%$ of children between the ages of 12 - 15 months continued breastfeeding [3]. Abuidhail et al. [6] reported that although postpartum mothers in Jordan initiated exclusive breastfeeding within the first hour of delivery, this practice declined gradually as the infant grow up within the first six months after birth.

Attitude toward breastfeeding among Jordanian women was recently studied by Al-Akour et al. [7] who reported that about $76.2 \%$ of Jordanian mothers were intended to breastfeed their infants. Further, Sunaa [8] found that the percentage of Jordanian infants who received breast milk was $76 \%$. Of those, $36 \%$ were exclusively breastfed. Jordanian researchers found that mothers who had a more positive attitude toward breastfeeding, mothers with previous experience in breastfeeding, and mothers who received husbands' support were more intended to breastfeed their infants [7]. In addition, Jordanian working mothers [8] [9], highly educated mothers [8] and those who delivered by cesarean section [9] were less likely to do breastfeeding. "Insufficient mother's milk" was the main cause of weaning for employed mothers [10].

Previous studies conducted in Jordan have focused on the association between breastfeeding attitudes, practices, and maternal characteristics. However, there is no sufficient evidence about factors related to infants' characteristics such as (infant gender, birth weight, gestational age, admission to neonatal intensive care unit, health condition, birth order, planned pregnancy and the type of delivery) on attitudes toward breastfeeding. In addition, there is a lack of studies about the association between infants' characteristics and the attitudes and practices of breastfeeding in general.

Therefore, this study aims to assess Jordanian mothers' attitudes toward breastfeeding in the light of infants' characteristics.

This study answers the following questions:

What are the attitudes that Jordanian mothers have toward breastfeeding? Are there any association between infants' characteristics and mothers' attitudes toward breastfeeding?

Understanding these factors may help health care professionals and policy makers to plan interventions and programs aiming to promote early initiation and maintaining of optimal breastfeeding practices. This is ultimately congruent with healthy people 2020 goal "to increase the proportion of infants who are breastfed" [11].

\section{Methods}

\subsection{Ethical Considerations}

Permissions to conduct this study were obtained from Zarqa University ethical committee in addition to the ethical committee at the Ministry Of Heath where the data were collected. Informed consent was obtained from 
participants after explaining the purpose of the study. Participation was voluntary and participants were assured about the anonymity and confidentiality of their personal data.

\subsection{Design, Setting and Sample}

A descriptive cross-sectional design was used in this study. A convenience sample of one hundred and thirty mothers recruited from four major governmental maternal-child health centers at Zarqa city-Jordan was participated in this study. All participants were Jordanian mothers, aged 18 years and above, had an infant since 1 year or less, had no medical problems during pregnancy, had no chronic medical problems, and able to read and speak Arabic. A self-administered questionnaire was used to collect information about infants' characteristics including infant gender, birth weight, gestational age, admission to neonatal intensive care unit, health condition, birth order, planned pregnancy and the type of delivery. The Iowa Infant Feeding Attitude Scale (IIFAS) was used to measure Jordanian mothers' attitudes toward breastfeeding. IIFAS is a valid and reliable measure that evaluates breastfeeding knowledge and attitudes cross-culturally [7] [12]-[14]. Higher scores indicate more positive attitudes toward breastfeeding.

The scale is self-administered and comprises 17 items rated on a five-point Likert scale ranged from $1=$ strongly disagree to $5=$ strongly agree. Eight items indicate positive attitudes to breastfeeding while nine items indicate positive attitudes to formula feeding. Items that reflect positive attitudes to formula feeding were scored reversely and the scores for each item are then summed. The total scores range from 17 which reflect the highest negative attitudes toward breastfeeding to 85 which indicate the highest positive attitude toward breastfeeding [14].

Translation of the scale from English to Arabic was done by the researcher and two maternity nursing academics and checked by a bilingual expert. Back-translation from Arabic to English was also conducted by another bilingual expert to ensure the accuracy of translation.

\subsection{Procedure}

Before data collection, a pilot study of 20 Jordanian women was conducted to check clarity of the questionnaire. Then, the researcher interviewed Jordanian mothers who attended the maternal-child health centers and explained to them the purposes of the study. Mothers who agreed to participate in the study and met the eligible criteria have signed the consent form and then filled the infants' characteristics sheet and a demographic data sheet that includes the mother's age, educational level, employment, parity, and previous experience in breastfeeding. Filling the questionnaires in the maternal-child health centers by mothers were conducted in the presence of the researcher. Participants were encouraged to ask questions or seek any clarification.

\subsection{Data Analysis}

Data were entered and analyzed using the Statistical Package for Social Sciences software version 17.0. Descriptive statistics were used to organize and summarize the study variables and each item in the questionnaire. A binary logistic regression (BLR) analysis was performed to determine whether the attitudes toward breastfeeding among Jordanian women are influenced by infants' characteristics. A p-value of 0.05 or less was considered statistically significant. For the purposes of binary logistic regression analysis, mothers were divided into two groups: those with an IIFAS score equals 51 or more, and those with a score less than 51.

\section{Results}

Data from 130 Jordanian women were included in the analysis. The age of participants ranged from (20 - 44) years. The mean age (SD) of participants were 26.7 (4.5). The majority of women were not employed (75\%), and had only high school education (67\%). Almost one third of women were smokers (31\%), and about (42.8\%) had family income of more than 250 USD. All women were living with their husbands (100\%). Regarding infants' characteristics, the percentage of male to female infants was equal (50\%). Among those, $80.8 \%$ of infants were weighted $2500 \mathrm{gm}$ and more. While $19.2 \%$ were less than $2500 \mathrm{gm}$. The majority of infants (78.5\%) were born full term at 37 weeks or more, and $21.5 \%$ were less than 37 weeks. Among those infants, $23.1 \%$ were admitted to neonatal intensive care unit and $76.9 \%$ did not admit to neonatal intensive care unit. Most infants were healthy (81.5\%) while $18.5 \%$ of infants had medical problems including jaundice, chest infection, and congenital 
abnormalities. Almost one third of infants were the first kid in their families (33.8\%) and (66.2\%) were not the first. Planned pregnancy was higher among this sample (66.2\%) while (33.8\%) of pregnancies were not planned. The majority of infants were born by normal vaginal delivery (66.9\%), and (33.1\%) were born by cesarean section Table 1.

In general, the IIFAS scores for women in this study indicate more positive attitudes toward breastfeeding, mean (SD) of the total scores was 63.5 (4.67). The items that scored the highest are "Breast milk is less expensive than formula" with a mean value of 4.74 and "Breast milk is more easily digested than formula" with a mean value of 4.62, while, the item "A mother who occasionally drinks alcohol, should not breastfeed her baby" with a mean value of 1.97 and the item "Mothers who formula-fed are miss out one of the great joys of motherhood" with a mean value of 2.73 are scored the lowest. Table 2 shows Jordanian mothers attitudes towards breastfeeding as measured by Iowa Infant Feeding Attitude Scale.

Table 3 shows the results of BLR model for predicting of breastfeeding from infants characteristics. According to the model, four variables were statistically significant contributing to the attitudes toward breastfeeding (gestational age, admission to neonatal intensive care unit, health status, and type of delivery).

Positive attitudes toward breastfeeding were 0.54 time higher among women who delivered by normal vaginal delivery than those who have cesarean section $(\mathrm{p}=0.040)$. In addition, women who had healthy infants were eight times more likely to have positive attitudes toward breastfeeding compared to women with ill infants $(\mathrm{p}=$

\begin{tabular}{|c|c|c|}
\hline Variables & $\mathrm{n}$ & $\%$ \\
\hline \multicolumn{3}{|l|}{ Gender } \\
\hline Male & 65 & 50 \\
\hline Female & 65 & 50 \\
\hline \multicolumn{3}{|l|}{ Birth weight } \\
\hline$\geq 2500 \mathrm{gm}$ & 105 & 80.8 \\
\hline$<2500$ gm & 25 & 19.2 \\
\hline \multicolumn{3}{|l|}{ Gestational age } \\
\hline$\geq 37$ weeks (full term infant) & 102 & 78.5 \\
\hline <37 weeks (preterm infant) & 28 & 21.5 \\
\hline \multicolumn{3}{|l|}{ Admission to NICU } \\
\hline Yes & 30 & 23.1 \\
\hline No & 100 & 76.9 \\
\hline \multicolumn{3}{|l|}{ Health status } \\
\hline Healthy & 106 & 81.5 \\
\hline Has a medical problem & 24 & 18.5 \\
\hline \multicolumn{3}{|l|}{ Birth order } \\
\hline First infant & 44 & 33.8 \\
\hline Others & 86 & 66.2 \\
\hline \multicolumn{3}{|l|}{ Planned pregnancy } \\
\hline Yes & 86 & 66.2 \\
\hline No & 44 & 33.8 \\
\hline \multicolumn{3}{|l|}{ Type of delivery } \\
\hline Normal vaginal delivery & 87 & 66.9 \\
\hline Cesarean section & 43 & 33.1 \\
\hline
\end{tabular}


Table 2. Attitudes towards breastfeeding among Jordanian mothers, measured by Iowa Infant Feeding Attitude Scale.

Attitudes towards breastfeeding $\quad$ Total $(\mathrm{n}=130)$

1. The nutritional benefits of breast milk last only until the baby is weaned from breast milk.

Mean

2. Formula-feeding is more convenient than breast-feeding.

3. Breast-feeding increases mother-infant bonding.

3.87

4. Breast milk is lacking in iron.

4.54

5. Formula-fed babies are more likely to be overfed than are breast-fed babies.

3.86

6. Formula-feeding is the better choice if a mother plans to work outside the home.

7. Mothers who formula-fed are miss out one of the great joys of motherhood.

3.73

8. Mothers should not breast-feed in public places such as restaurants.

9. Babies fed breast milk are healthier than babies who are fed formula.

10. Breast-fed babies are more likely to be overfed than are formula-fed babies.

11. Fathers feel left-out if a mother breastfeeds.

12. Breast milk is the ideal food for babies.

13. Breast milk is more easily digested than formula.

14. Formula is as healthy for an infant as breast milk.

15. Breast-feeding is more convenient than formula feeding.

16. Breast milk is less expensive than formula.

17. A mother who occasionally drinks alcohol, should not breastfeed her baby.

\section{Table 3. Results of BLR model.}

\begin{tabular}{lcccc}
\hline & B & Wald & Sig. & Exp (B) \\
\hline Gender (1) & -16.694 & 0.000 & 0.994 & 0.000 \\
Weight (1) & 25.877 & 0.000 & 0.991 & $1.246 \mathrm{E} 22$ \\
Gestational age (1) & 2.224 & 7.425 & 0.013 & 0.131 \\
Admission (1) & -1.569 & 0.007 & 0.043 & 0.208 \\
Health status (1) & 1.846 & 5.487 & 0.021 & 8.113 \\
Birth order (1) & 0.756 & 0.145 & 0.701 & 2.130 \\
Planned pregnancy (1) & -33.330 & 1.001 & 0.693 & 0.000 \\
Type of delivery (1) & 4.259 & 6.110 & 0.040 & 0.547 \\
\hline
\end{tabular}

0.021). However, women who delivered preterm infants were 0.13 time less likely to have positive attitudes toward breastfeeding than women who delivered full term infants $(p=0.013)$. Similarly, women whose infants admitted to neonatal intensive care unit were 0.20 time less likely to have positive attitudes toward breastfeeding than those with healthy neonates $(\mathrm{p}=0.043)$.

\section{Discussion}

This study aimed to assess Jordanian mothers' attitudes toward breastfeeding and determine any association between attitudes toward breastfeeding and infants' characteristics. The results revealed that Jordanian mothers have positive attitudes toward breastfeeding. Previous study showed that Jordanian women with positive attitudes 
to breastfeeding are more intended to breastfeed their children [7]. Similarly, Persad and Mensinger [15] found that the intent for breastfeeding was associated with positive breastfeeding attitudes. A study conducted by Shaker et al. [16] found that parents who breastfed their infants had more positive attitudes toward breastfeeding than parents who used formula feeding. In addition, a positive attitude toward breastfeeding was associated with a longer duration of breastfeeding [17]. This suggests that parental attitudes toward infant's feeding are predictors of the choice and duration of infant's feeding. Therefore, nurses should assess and understand mothers' attitudes toward infants feeding and consequently design appropriate interventions to enhance breastfeeding practices.

The present study identified four factors significantly associated with attitudes toward breastfeeding. Vaginal delivery was positively associated with attitudes toward breastfeeding compared to cesarean delivery. This might be due to the pain, discomfort and fatigue associated with cesarean section that prevent mothers from the initiation of breastfeeding. A study conducted in Canada by Al-Sahab et al. [18] revealed that vaginal birth increased the rates of exclusive breastfeeding by $25 \%$ compared to cesarean birth. Similarly, Grossman et al. [19] found negative association between cesarean birth and the duration of breastfeeding. In contrast, Al-Ghwass and Ahmed [20] and Chye [21] found no significant association between the mode of delivery and exclusive breastfeeding.

In this study, gestational age was another factor that could affect mothers' attitudes toward breastfeeding. Full term infant was positively associated with attitudes toward breastfeeding. This might be justified by the high self-confidence that mother has as the infant is fully mature and able to do effective sucking. Breastfeeding self-efficacy is found to be a significant predictor of breastfeeding duration and exclusivity [22] [23]. This result is in agreement with a study conducted by Tan [24] in Malaysia whereby mothers with full-term infants were twice as likely as to exclusively breastfeed compared to mothers with preterm infants.

Admission to neonatal intensive care unit and health status of infants were other factors that could affect mothers' attitudes toward breastfeeding. Jordanian mothers with healthy infants as well as mothers whose infants did not admit to neonatal intensive care unit had positive attitudes to breastfeeding. This might be because of the mothers' fear and low confidence to deal with their ill infants. In addition, early separation of mothers and their ill infants may impede the initiation of breastfeeding. The result of the present study is in agreement with a study conducted by Al-Sahab et al. [18] who found that mothers who had their infants admitted to neonatal intensive care unit after birth were less likely to practice exclusive breastfeeding. Jakobsen $e t$ al. [25] claimed that child illness is strongly associated with early weaning from breastfeeding.

With regard to infant's gender there was no significant association with the attitudes to breastfeeding. Contrary to that, a study conducted by Al-Ghwass and Ahmed [20] revealed that infant's gender was a significant predictor associated with exclusive breastfeeding. Males were (1.8) times more likely to be exclusively breastfed than females.

However, the result of the present study is in agreement with a study conducted by Al-Ghwass and Ahmed [20] who found no association between the birth weight and exclusive breastfeeding.

The results of this study also revealed that there was no significant association between birth order and attitudes toward breastfeeding. Studies regarding the association between birth order and breastfeeding are inconsistent. Al-Ghwass and Ahmed [20] found no association between exclusive breastfeeding and the number of deliveries. In contrast, a study conducted by Tan et al. [24] revealed that exclusive breastfeeding was more common among mothers with more than one child. Similarly, Wojdan-Godek et al. [26] reported that the first child in the family was associated with cessation of exclusive breastfeeding in Poland. Al-Sahab et al. [18] found that mothers who had more pregnancies had an increased likelihood of breastfeeding exclusively for six months. Mothers with more than one child are suggested to have increased knowledge, skills and experiences from previous pregnancies.

With regard to planned pregnancy there was no significant association with attitudes toward breastfeeding. In contrast, a study conducted by Taylor and Cabral [27] in the united states revealed that white women who intended to become pregnant were more likely to initiate and continue breastfeeding than women who did not intend to become pregnant.

The findings of this study add to the literature the concept of infants' characteristics and its impact on attitudes toward breastfeeding. Health care professionals and policy makers in Jordan can utilize the findings of this study to develop programs on promoting breastfeeding. Future studies may include qualitative parts to support this under-studied phenomenon. 


\section{Limitation}

This study was limited to Jordanian women who live in Zarqa city. This may limit the generalizability of the study findings to all Jordanian mothers due to non-investigated geographical and socioeconomic variables.

\section{Conclusion}

Breastfeeding is agreed to be the desired method of infants' feeding. Jordanian mothers have positive attitudes toward breastfeeding. Factors associated with breastfeeding attitudes were: women who delivered full term infants; women who have healthy infants; and women who delivered by normal vaginal delivery. Admission to neonatal intensive care unit was negatively associated with attitudes toward breastfeeding. These factors should be considered when designing programs that encourage breastfeeding.

\section{Competing Interests}

The author declares that she has no competing interests.

\section{Acknowledgements}

The author would like to thank the study participants as well as health care professionals at the maternal-child health centers in Zarqa city/Jordan.

\section{Funding Statement}

"This research is funded by the Deanship of Research and Graduate Studies in Zarqa University /Jordan”.

\section{References}

[1] UNICEF (2014) Breastfeeding. http://www.unicef.org/nutrition/index_24824.html

[2] World Health Organization (2013) 10 Facts on Breastfeeding. http://www.who.int/features/factfiles/breastfeeding/en/

[3] Jordan Department of Statistics (2012) Jordan Population and Family Health Survey, Infant Feeding Practices.

[4] The Quran, the Holy Book of Muslims, Al Baqarah 233.

[5] American Academy of Pediatrics (2005) Breastfeeding and the Use of Human Milk. Pediatrics, 115, 496-506. http://dx.doi.org/10.1542/peds.2004-2491

[6] Abuidhail, J., Al-Modallal, H., Yousif, R. and Almresi, N. (2013) Exclusive Breast Feeding (EBF) in Jordan: Prevalence, Duration, Practices, and Barriers. Midwifery, 30, 331-337. http://dx.doi.org/10.1016/j.midw.2013.01.005

[7] Al-Akour, N., Khassawneh, M., Khader, Y., Ababneh, A. and Haddad, A. (2010) Factors Affecting Intention to Breastfeed among Syrian and Jordanian Mothers: A Comparative Cross-Sectional Study. International Breastfeeding Journal, 5, 6. http://dx.doi.org/10.1186/1746-4358-5-6

[8] Sunna, L. (2012) Child Feeding Patterns and Diarrhea. Bahrain Medical Bulletin, 34, 180-185.

[9] Khassawneh, M., Khader, Y., Amarin, Z. and Alkafajei, A. (2006) Knowledge, Attitude and Practice of Breastfeeding in the North of Jordan: A Cross Sectional Study. International Breastfeeding Journal, 1, 17. http://dx.doi.org/10.1186/1746-4358-1-17

[10] Mubaideen, M.M. and Al-Saraireh, R.M. (2006) Breast-Feeding Pattern in Selected Antenatal Care Clinics in Jordan. JRMS, 13, 74-78.

[11] United States Department of Health and Human Services (2010) Healthy People 2020 Topics \& Objectives. http://www.usbreastfeeding.org/LegislationPolicy/FederalPoliciesInitiatives/HealthyPeople2020BreastfeedingObjectiv es/tabid/120/Default.aspx

[12] Al-Madani, M., Vydelingum, V. and Lawrence, J. (2010) Saudi Mothers’ Expected Intentions and Attitudes toward Breast-Feeding. ICAN: Infant, Child, \& Adolescent Nutrition, 2, 187-198. http://dx.doi.org/10.1177/1941406410369699

[13] Sittlington, J., Stewart-Knox, B., Wright, M., Bradbury, I. and Scott, J. (2007) Infant-Feeding Attitude of Expectant Mothers in Northern Ireland. Health Education Research, 22, 561-570. http://dx.doi.org/10.1093/her/cyl113

[14] De la Mora, A., Russell, D.W., Dungy, C.I., Losch, M. and Dusdieker, L. (1999) The Iowa Infant Feeding Attitude Scale: Analysis of Reliability and Validity. Journal of Applied Social Psychology, 29, 2362-2380.

http://dx.doi.org/10.1111/j.1559-1816.1999.tb00115.x 
[15] Persad, M.D. and Mensinger, J.L. (2008) Maternal Breastfeeding Attitudes: Association with Breastfeeding Intent and Socio-Demographics among Urban Primiparas. Journal of Community Health, 33, 53-60. http://dx.doi.org/10.1007/s10900-007-9068-2

[16] Shaker, I., Scott, J.A. and Reid, M. (2004) Infant Feeding Attitudes of Expectant Parents: Breastfeeding and Formula Feeding. Journal of Advanced Nursing, 45, 260-268. http://dx.doi.org/10.1046/j.1365-2648.2003.02887.x

[17] Brown, A. and Lee, M. (2011) An Exploration of the Attitudes and Experiences of Mothers in the United Kingdom Who Chose to Breastfeed Exclusively for 6 Months Postpartum. Breastfeeding Medicine, 6, 197-204. http://dx.doi.org/10.1089/bfm.2010.0097

[18] Al-Sahab, B., Lanes, A., Feldman, M. and Tamim, H. (2010) Prevalence and Predictors of 6-Month Exclusive Breastfeeding among Canadian Women: A National Survey. Pediatrics, 10, 20-29.

[19] Grossman, L.K., Fitzsimmons, S.M., Larsen-Alexander, J.B., Sachs, L. and Harter, C. (1990) The Infant Feeding Decision in Low and Upper Income Women. Clinical Pediatrics, 29, 30-37. http://dx.doi.org/10.1177/000992289002900105

[20] Al Ghwass, M. and Ahmed, D. (2011) Prevalence and Predictors of 6-Month Exclusive Breastfeeding in a Rural Area in Egypt. Breastfeeding Medicine, 6, 191-196. http://dx.doi.org/10.1089/bfm.2011.0035

[21] Chye, J.K., Zain, Z., Lim, W.L. and Lim, C.T. (1997) Breastfeeding at 6 Weeks and Predictive Factors. Journal of Tropical Pediatrics, 43, 287-292. http://dx.doi.org/10.1093/tropej/43.5.287

[22] Blyth, R., Creedy, D.K., Dennis, C.-L., Moyle, W., Pratt, J. and De Vries, M. (2002) Effect of Maternal Confidence on Breastfeeding Duration: An Application of Breastfeeding Self-Efficacy Theory. Birth, 29, 278-284. http://dx.doi.org/10.1046/j.1523-536X.2002.00202.x

[23] Nichols, J., Schutte, N.S., Brown, R.F., Dennis, C.-S. and Price, I. (2009) The Impact of a Self-Efficacy Intervention on Short-Term Breastfeeding Outcomes. Health Education \& Behavior, 36, 250-258. http://dx.doi.org/10.1177/1090198107303362

[24] Tan, K.L. (2011) Factors Associated With Exclusive Breastfeeding among Infants under Six Months of Age in Peninsular Malaysia. International Breastfeeding Journal, 6, 2. http://dx.doi.org/10.1186/1746-4358-6-2

[25] Jakobsen, M.S., Sodemann, M., Molbak, K. and Aaby, P. (1996) Reason for Termination of Breastfeeding and the Length of Breastfeeding. International Journal of Epidemiology, 25, 115-121. http://dx.doi.org/10.1093/ije/25.1.115

[26] Wojdan-Godek, E., Mikiel-Kostyra, K. and Mazur, J. (2000) Factors Associated with Exclusive Breastfeeding of Infants in Poland. Medycyna Wieku Rozwojowego, 4, 15-24. (In Polish)

[27] Taylor, J.S. and Cabral, H.J. (2002) Are Women with an Unintended Pregnancy Less Likely to Breastfeed? The Journal of Family Practice, 51, 431-436. 